\title{
Actinorhizal Signaling Molecules: Frankia Root Hair Deforming Factor Shares Properties With NIN Inducing Factor
}

OPEN ACCESS

Edited by:

Ulrike Mathesius,

Australian National University,

Australia

Reviewed by:

Dugald Reid,

Aarhus University, Denmark

Ton Bisseling,

Wageningen University \& Research,

Netherlands

*Correspondence:

Sergio Svistoonoff

sergio.svistoonoff@ird.fr

Specialty section:

This article was submitted to Plant Evolution and Development,

a section of the journal

Frontiers in Plant Science

Received: 31 May 2018 Accepted: 25 September 2018

Published: 18 October 2018

Citation:

Cissoko M, Hocher V, Gherbi H, Gully D, Carré-Mlouka A, Sane S, Pignoly S, Champion A, Ngom M,

Pujic P, Fournier P, Gtari M,

Swanson E, Pesce C, Tisa LS, Sy MO and Svistoonoff S (2018) Actinorhizal

Signaling Molecules: Frankia Root

Hair Deforming Factor Shares

Properties With NIN Inducing Factor.

Front. Plant Sci. 9:1494.

doi: 10.3389/fpls.2018.01494
Maimouna Cissoko ${ }^{1,2,3,4}$, Valérie Hocher ${ }^{4}$, Hassen Gherbi ${ }^{4}$, Djamel Gully 4 , Alyssa Carré-Mlouka, ${ }^{4,5}$, Seyni Sane ${ }^{6}$, Sarah Pignoly 1,2,4, Antony Champion 1,2,7, Mariama Ngom 1,2, Petar Pujic ${ }^{8}$, Pascale Fournier ${ }^{8}$, Maher Gtari ${ }^{9}$, Erik Swanson ${ }^{10}$, Céline Pesce ${ }^{10}$, Louis S. Tisa ${ }^{10}$, Mame Oureye Sy ${ }^{3}$ and Sergio Svistoonoff $1,2,4 *$

\begin{abstract}
${ }^{1}$ Laboratoire Commun de Microbiologie, Institut de Recherche pour le Développement/Institut Sénégalais de Recherches Agricoles/Université Cheikh Anta Diop, Centre de Recherche de Bel Air, Dakar, Senegal, ${ }^{2}$ Laboratoire Mixte International Adaptation des Plantes et Microorganismes Associés Aux Stress Environnementaux, Centre de Recherche de Bel Air, Dakar, Senegal, ${ }^{3}$ Laboratoire Campus de Biotechnologies Végétales, Département de Biologie Végétale, Faculté des Sciences et Techniques, Université Cheikh Anta Diop, Dakar, Senegal, ${ }^{4}$ Laboratoire des Symbioses Tropicales et Méditerranéennes, Institut de Recherche pour le Développement/INRA/CIRAD, Université Montpellier/SupAgro, Montpellier, France, ${ }^{5}$ UMR 7245, Molécules de Communication et Adaptation des Microorganismes, Muséum National d'Histoire Naturelle, Centre National de la Recherche Scientifique, Sorbonne Universités, Paris, France, ${ }^{6}$ Laboratoire de Botanique et de Biodiversité Végétale, Département de Biologie Végétale, Faculté des Sciences et Techniques, Université Cheikh Anta Diop, Dakar, Senegal, ${ }^{7}$ UMR Diversité Adaptation et Développement des Plantes (DIADE), Institut de Recherche pour le Développement, Montpellier, France, ${ }^{8}$ Ecologie Microbienne, UMR 5557 CNRS, Université Lyon 1, Villeurbanne, France, ${ }^{9}$ Institut National des Sciences Appliquées et de Technologie, Université Carthage, Tunis, Tunisia, ${ }^{10}$ Department of Molecular, Cellular, and Biomedical Sciences, University of New Hampshire, Durham, NH, United States
\end{abstract}

Actinorhizal plants are able to establish a symbiotic relationship with Frankia bacteria leading to the formation of root nodules. The symbiotic interaction starts with the exchange of symbiotic signals in the soil between the plant and the bacteria. This molecular dialog involves signaling molecules that are responsible for the specific recognition of the plant host and its endosymbiont. Here we studied two factors potentially involved in signaling between Frankia casuarinae and its actinorhizal host Casuarina glauca: (1) the Root Hair Deforming Factor (CgRHDF) detected using a test based on the characteristic deformation of $C$. glauca root hairs inoculated with F. casuarinae and (2) a NIN activating factor (CgNINA) which is able to activate the expression of CgNIN, a symbiotic gene expressed during preinfection stages of root hair development. We showed that CgRHDF and CgNINA corresponded to small thermoresistant molecules. Both factors were also hydrophilic and resistant to a chitinase digestion indicating structural differences from rhizobial Nod factors (NFs) or mycorrhizal Myc-LCOs. We also investigated the presence of CgNINA and CgRHDF in 16 Frankia strains representative of Frankia diversity. High levels of root hair deformation $(\mathrm{RHD})$ and activation of ProCgNIN were detected for Casuarina-infective strains from clade IC and closely related strains from clade la unable to nodulate C. glauca. Lower levels were present for distantly related strains belonging to clade III. No CgRHDF or CgNINA could be detected for Frankia coriariae (Clade II) or for uninfective strains from clade IV.

Keywords: symbioses, nodulation factors, nodule inception, Casuarina, Alnus, Discaria 


\section{INTRODUCTION}

Legumes and actinorhizal plants form a $\mathrm{N}_{2}$-fixing root nodule symbiosis in association with rhizobia and Frankia bacteria, respectively (Vessey et al., 2005). The establishment of these beneficial bacterial-plant relationships requires communication between the partners. Rhizobial symbioses have received considerable attention because several legumes are important crop species. However, actinorhizal symbioses, which play an important ecological role (Dawson, 2008), have been less well studied and the molecular dialog between Frankia and their host plants is still poorly understood. One reason is that most actinorhizal plants are woody shrubs or trees for which genetic approaches are very difficult (Wall, 2000; Perrine-Walker et al., 2011). In addition, the genetics of the bacterial partner, Frankia, is not fully developed and up to now Frankia cells remain recalcitrant to stable genetic transformation (Kucho et al., 2009, 2017). Recent progress including the sequencing of several Frankia genomes (Normand et al., 2007; Tisa et al., 2016), transcriptomic studies (Alloisio et al., 2010; Benson et al., 2011), proteomic studies (Mastronunzio and Benson, 2010; Ktari et al., 2017) together with functional studies on several actinorhizal species (Svistoonoff et al., 2014) have opened new avenues for identifying components involved in the initial symbiotic dialog between the two partners.

The interaction of rhizobia with model legumes begins with the production and recognition of signal molecules by their respective eukaryotic and prokaryotic symbiotic partners (Oldroyd, 2013). Early events leading to nodule formation involve bacterial penetration into their hosts via root hairs. Bacteria elicit the stimulation and reorientation of root hair cell wall growth. This rhizobia-induced tip growth results first in the entrapment of the bacteria within curled root hairs and then in the initiation and development of infection threads (ITs), tubular structures through which bacteria pass on their way down the root hair and into the underlying cortical cell layers (Lhuissier et al., 2001). Ahead of the advancing threads, cells in the inner cortex are induced to dedifferentiate and divide, and a nodule primordium is formed. In the first part of the signal exchange, the plant roots secrete flavonoids that lead to the activation of a set of rhizobial genes (the nod genes), which are essential for infection, nodule development and the control of host specificity (Masson-Boivin et al., 2009; Oldroyd, 2013). These genes are responsible for the synthesis of lipo-chito-oligosaccharides (LCOs) called Nod factors (NFs) that signal back to the plant (Oldroyd, 2013). NF biosynthesis is dependent on nodABC genes which are present in all rhizobia able to synthetize NFs and strain-specific combinations of other nodulation genes responsible for the addition of various decorations to the core structure. (Masson-Boivin et al., 2009). In model legumes, NFs perception elicits a range of responses including ion fluxes, calcium oscillations, changes in gene expression patterns, and extensive deformation of roots hairs, which has been used as a bioassay to identify the chemical nature of NFs (Lerouge et al., 1990; Oldroyd, 2013).
Much less is known about signaling molecules involved in the actinorhizal symbioses. Canonical nod $A B C$ genes are not found in the sequenced genomes of 36 Frankia strains including Frankia alni and Frankia casuarinae (Tisa et al., 2016) confirming a previous report showing that $F$. alni DNA will not complement rhizobial nod mutants (Cérémonie et al., 1998). Only distant homologs of $\operatorname{nodB}$ and nodC are found in F. alni genome. Unlike rhizobial nod genes, they are not organized into a cluster together with other symbiotic genes and their expression is not induced under symbiotic conditions (Normand et al., 2007; Alloisio et al., 2010). These findings are consistent with experiments showing that chitin oligomers similar to rhizobial NFs are not be detected in F. alni culture supernatant (Cérémonie et al., 1999) suggesting structural differences between the Frankia symbiotic signals and rhizobial NFs. Recently, canonical nodABC genes have been found in the genome of two uncultured Frankia strains: Candidatus Frankia datiscae Dg1 and Candidatus Frankia californicae Dg2 (Persson et al., 2015; Nguyen et al., 2016), and in one isolated strain, Frankia sp. NRRL B-16219 (Ktari et al., 2017). F. datiscae Dg1 nodABC genes are arranged in two operons which are expressed in Datisca glomerata nodules, but their involvement in symbiotic signaling is still not known (Persson et al., 2015).

Frankia is able to infect their host root either through intracellular (root hair) or intercellular modes. In the first case, one of the earliest visible plant response to Frankia is an extensive deformation of root hairs. This response occurs in actinorhizal plants belonging to the order Fagales (Betulaceae, Casuarinaceae) that display a range of relatively advanced features reminiscent of model legumes: a complex root hair infection process involving the formation of ITs and the implication of cortical cell divisions at the initial stages of infection (Svistoonoff et al., 2014). Frankia culture supernatants also cause root hair deformation (RHD) and a Frankia root hair deforming factor in Alnus (AgRHDF) was identified (Prin and Rougier, 1987; Ghelue et al., 1997; Cérémonie et al., 1999; Gabbarini and Wall, 2011). Using RHD as a bioassay, partial purification was achieved. AgRHDF is a relatively small $(<3 \mathrm{kDa})$, heat stable, hydrophilic molecule that is resistant to a chitinase treatment, but its chemical structure remains unknown (Cérémonie et al., 1999).

In recent years, we have developed complementary bioassays using plant genes that are specifically expressed in response to interaction with a compatible Frankia. This approach is particularly well suited for C. glauca where transgenic plants containing promoters of symbiotic genes fused to either GUS or GFP can be generated (Svistoonoff et al., 2010a). Expressed Sequence Tag (EST) libraries of C. glauca and Alnus glutinosa (Hocher et al., 2006, 2011) provide extensive lists of genes potentially involved in the actinorhizal symbiosis. Among the candidate genes, we identified CgNIN, the putative ortholog of legume NIN genes, which encodes a transcription factor playing a central role in rhizobial nodulation (Schauser et al., 1999; Marsh et al., 2007; Soyano et al., 2013, 2014; Yoro et al., 2014). In C. glauca, CgNIN also has an important role in nodulation particularly at early steps of infection (Clavijo et al., 2015). After contact with either Frankia cells or cellfree Frankia supernatants, the CgNIN promoter is strongly 
activated at 12 to $48 \mathrm{~h}$ (Clavijo et al., 2015). This property was used to establish a new bioassay leading to the partial purification and characterization of a NIN activating factor, called CgNINA. While rhizobial NFs are amphiphilic chitinbased molecules, CgNINA, like AgRHDF, is hydrophilic and resistant to chitinase (Chabaud et al., 2016). However, it is not known to what extent CgNINA is related to factors able to deform root hairs.

Further experiments concerning these Frankia symbiotic factors are reported here. We show that C. glauca was able to perceive a root hair deforming factor secreted by $F$. casuarinae (CgRHDF) and the properties of CgRHDF were compared to those previously identified with CgNINA. The presence of CgNINA and CgRHDF in strains representative of Frankia diversity was investigated.

\section{MATERIALS AND METHODS}

\section{Plant Material and Growth Conditions}

Casuarina glauca seeds (seed lot 15.934, ref.086-5929) were provided by the Australian Tree Seed Centre ${ }^{1}$. Ochetophila trinervis (= Discaria trinervis) seeds were collected from plants growing in Pampa de Huenuleo (Bariloche, Argentina). A. glutinosa seeds were harvested from a tree situated in the left bank of Rhône River in Lyon, France. C. glauca and $O$. trinervis seeds were disinfected and germinated in a sterilized substrate for three weeks and transferred into glass tubes filled with a modified Broughton and Dilworth (BD) medium as described previously (Ngom et al., 2016). A. glutinosa seeds were washed in distilled sterile water for $30 \mathrm{~min}$ before sterilization in $96 \%$ ethanol for 30 min followed by $3 \%$ solution of calcium hypochlorite for $30 \mathrm{~min}$. Seeds were germinated on $1.5 \%$ plant agar for 10 days at $20^{\circ} \mathrm{C}$, and transferred in $5 \mathrm{~mL}$ tubes containing liquid Fahraeus medium without nitrogen (Fahraeus, 1957). Plants were grown for 6 weeks in growth chamber at $25^{\circ} \mathrm{C}$ at $75 \%$ relative air humidity and 16 h light cycle/day. Transgenic C. glauca plants containing a ProCgNIN:GFP construct described previously (Clavijo et al., 2015) were grown in hydroponics in pots containing the modified BD medium and vegetatively propagated as described previously (Svistoonoff et al., 2010b).

\section{Preparation of Cell-Free Supernatants and Inoculation}

The bacterial strains used in this study are listed in Supplementary Table S1 and were grown for twenty-one days in modified basic propionate (BAP) media described previously (Ngom et al., 2016) according to conditions listed in Supplementary Table S1. Bacterial cultures were exposed to plant root exudates (RE) for five days as described previously (Beauchemin et al., 2012; Clavijo et al., 2015; Chabaud et al., 2016). Cell-free supernatant fluids were purified from cultures showing an absorbance of 0.3 at $595 \mathrm{~nm}$. Cultures were collected by centrifugation at $4,000 \mathrm{~g}$ for $5 \mathrm{~min}$ and the supernatant

${ }^{1}$ https://www.csiro.au/en/Research/Collections/ATSC fluids were filtered through a $0.22 \mu \mathrm{m}$ filter as described in Chabaud et al., 2016. Unless otherwise indicated, experiments were performed with the supernatant fluids of a $F$. casuarinae culture induced with RE from its host plant C. glauca and referred to as FCS for Frankia casuarinae supernatant. FCS were concentrated fifty times (FCS 50X) using an R-210/215 evaporator (BÜCHI Labortechnik AG, Switzerland). Nodulation experiments with the different Frankia strains were performed as described previously (Alloisio et al., 2010; Svistoonoff et al., 2010b; Imanishi et al., 2011).

\section{Characterization of $\boldsymbol{F}$. casuarinae Supernatant Fluids \\ Temperature and $\mathrm{pH}$ Sensitivity}

To test heat inactivation, FCS $50 \mathrm{X}$ was autoclaved at $120^{\circ} \mathrm{C}$ for $20 \mathrm{~min}$. Cold sensitivity was determined by freezing FCS at $80^{\circ} \mathrm{C}$ for $1 \mathrm{~h}$. The effects of $\mathrm{pH}$ on FCS activity were determined as follows: the initial $\mathrm{pH}$ of FCS (6.7) was adjusted to $\mathrm{pH} 3,5$, 7,8 or 10 by adding either $\mathrm{HCl}$ or $\mathrm{KOH}$ solutions. The FCS mixtures that these $\mathrm{pH}$ values were incubated for $1 \mathrm{~h}$ at room temperature and neutralized back to $\mathrm{pH} 6.7$ by adding either $\mathrm{HCl}$ or $\mathrm{KOH}$ solutions before performing the bioassays described below. Samples that lost CgNINA or CgRHDF activities were sonicated for 30 min using a Branson 2510 sonicator.

\section{Size Fractionation}

To estimate the size of signaling molecules, the FCS samples were dialyzed as described in Chabaud et al., 2016. Ten $\mathrm{mL}$ of FCS 50X were dialyzed for $12 \mathrm{~h}$ at $4^{\circ} \mathrm{C}$ against $5 \mathrm{~L}$ of ultrapure water with stirring and using either a 100-500, 5001000 or 3500-5000 Da cutoff membrane (Float-A-Lyzer G2 dialysis devices, Spectrum Laboratories, CA, United States). The dialyzed solutions were tested using the CgRHD and CgNINA bioassays described below. The size of active compounds was also estimated using centrifugal filters with cutoffs of 30,10, and $3 \mathrm{kDa}$ (Amicon Ultra-4 centrifugal filters; Merck-Millipore, Cork, Ireland). Four $\mathrm{mL}$ of $50 \mathrm{X}$ FCS were loaded on a $30 \mathrm{kDa}$ cell which was spun at $4,000 \mathrm{~g}$ for $20 \mathrm{~min}$. The filtrate recovered from the $30 \mathrm{kDa}$ filtration was treated similarly using a $10 \mathrm{kDa}$ cell, and the resulting $10 \mathrm{kDa}$ filtrate was added to a $3 \mathrm{kDa}$ Cell and spun.

\section{Phase Extraction and Sensitivity to Chitinase}

Two sequential butanol extractions were performed on FCS 50X with a ratio 1-butanol / water (1:3; v/v) described previously (Chabaud et al., 2016). The butanol phase was evaporated at $80^{\circ} \mathrm{C}$ under a nitrogen flow and the residue was dissolved in $20 \%$ acetonitrile as described in (Chabaud et al., 2016). Chitinase digestions were performed on the aqueous phase extract as described previously (Chabaud et al., 2016). Chitinase activity was assessed using a colorimetric method to estimate the amount of $\mathrm{p}$-nitrophenol ( $\mathrm{p}-\mathrm{NP}$ ) released from a reaction mixture containing the substrate $\mathrm{p}$-nitrophenyl $\mathrm{N}$-acetyl glucosaminide (p-NP-NAG) (Parham and Deng, 2000). A solution containing $1 \mathrm{mg} \mathrm{mL} \mathrm{mL}^{-1}$ of Streptomyces griseus chitinase (C6137; SigmaAldrich) in a $50 \mathrm{mM}$ phosphate buffer $\mathrm{pH} 6.0$ was prepared. A portion of this solution $(100 \mu \mathrm{l})$ was mixed with $50 \mu \mathrm{l}$ of $5 \mathrm{X}$ 
FCS aqueous extract, $100 \mu \mathrm{l}$ of p-NP-NAG solution at $10 \mathrm{mM}$ and $250 \mu \mathrm{l}$ of acetate buffer ( $\mathrm{pH} 5.50,1 \mathrm{M}$ ). The reaction was incubated at $37^{\circ} \mathrm{C}$ for $1 \mathrm{~h}$ under stirring and was terminated by the addition of $250 \mu \mathrm{l}$ of $\mathrm{CaCl}_{2}$ at $0.5 \mathrm{M}$ and $1000 \mu \mathrm{l}$ of $\mathrm{NaOH}$ at $0.5 \mathrm{M}$. The amount of $\mathrm{p}-\mathrm{NP}$ released was evaluated by measuring the absorbance at $400 \mathrm{~nm}$ with a spectrophotometer. Enzyme activity was expressed in $\mu \mathrm{g}$ of liberated $\mathrm{p}-\mathrm{NP}$ per hour of incubation. Control reactions were performed without $\mathrm{p}-\mathrm{NP}$ NAG, without chitinase and without the aqueous FCS extract.

\section{Root Hair Deformation and NINA Bioassays in Casuarina glauca}

Unless otherwise indicated, CgRHD and CgNINA bioassays were performed on aliquots of the same solution and the amount needed to achieve a final concentration equivalent a $10^{-2}$ dilution of raw (no diluted) Frankia culture supernatant was added to the nitrogen-free BD medium. All experiments were performed on at least 4 plants. At least two independent experiments were carried out for each tested solution.

The deformation of C. glauca root hairs (CgRHD) was evaluated using 3 week-old non transgenic plants grown in glass tubes exposed to nitrogen starvation for one week as described previously (Ngom et al., 2016). Treatments were performed by replacing the medium with fresh nitrogen-free BD medium containing the assayed solution. Deformation of root hairs situated on small lateral roots was scored as described by Clavijo et al. (2015) using micrographs taken with a BX50F microscope (Olympus) equipped with a Micro Publisher 3.3 RTV (Qimaging) digital camera. A blind evaluation of each micrograph was performed to determine the deformation level of observed root hairs using the following scale based on Clavijo et al. (2015): 0a: no deformation; 0b: straight root hair with tip swelling; 1 weak deformation, only one change in growth direction; 2 : intermediate deformation, more than one change in growth direction but no bifurcation; and 3: strong deformation: one or more bifurcations (Figure 1). Deformation levels 0a and ob were considered non-symbiotic. For each experiment at least four plants were analyzed per treatment and 6 small lateral roots were analyzed per plant. The total number of root hairs scored for each level of symbiotic deformation was used for the statistical analyzes described below. Each experiment was repeated four times independently.

For each treatment the percentage of symbiotic deformation (\%SyD) defined as the proportion of root hairs showing a symbiotic response was calculated and used to determine a deformation index using the following scale: level 1: SyD $<15 \%$; level 2: $16 \%<$ SyD < 25\%; level 3: $26 \%<$ SyD < 40\%; level 4: SyD $>41 \%$.

The activation of ProCgNIN in response to tested solutions was evaluated using transgenic ProCgNIN:GFP plants that were grown in hydroponics deprived of nitrogen for one week as previously described (Clavijo et al., 2015; Chabaud et al., 2016). After $24 \mathrm{~h}$ of contact with tested solutions, GFP fluorescence was monitored in the short lateral root hairs using an AZ100 macroscope (Nikon) equipped with a $5 \mathrm{X}$ objective, a GFP filter (Excitation filter $470 \mathrm{~nm} \pm 40 \mathrm{~nm}$; Barrier filter $535 \mathrm{~nm} \pm 50 \mathrm{~nm}$;
Nikon) and a digital camera Sight D5 RI1 (Nikon). For each observation, a blind evaluation of GFP fluorescence levels was performed using the following scale: 0: no detectable fluorescence; 1: weak fluorescence; 2: intermediate fluorescence; and 3: strong fluorescence (Figure 1). For each experiment the number of plants with a given fluorescence level was used for the statistical analysis described below. Each experiment was repeated at least four times independently.

\section{Root Hair Deformation Bioassay in A. glutinosa}

The deformation of A. glutinosa root hairs (AgRHD) was evaluated using 7 week-old plants that had at least four well developed secondary roots. Biological tests were performed at $10^{-2}$ final dilutions of Frankia culture supernatant fluids on plants growing in $5 \mathrm{ml}$ Fahraeus media without nitrogen. Evaluation of deformation was done in the region located about $1.5 \mathrm{~cm}$ from the root tip and five levels of RHD were recorded for each observed root: 0a: no deformation; 0b: swelling; 1 : branching; 2: branching and partial deformation; 3: total RHD and retracting. Deformation levels $0 \mathrm{a}$ and $0 \mathrm{~b}$ were considered as non-symbiotic. All experiments were performed on at least 3 plants and 5 roots were observed for each plant.

\section{Statistical Analyses}

Statistical analyses were performed on raw data: the number of hairs counted in each level of deformation using the $\mathrm{R}$ software package (R Core Team, 2013). A Shapiro-Wilk normality test was performed followed by a non-parametric Kruskal-Wallis multiple comparison test and a pairwise Wilcoxon test. These tests were used to compare the symbiotic response obtained for each treatment.

\section{Phylogenetic Tree}

The strict core genome of 17 Frankia strains was determined with the Get_Homologs package (Contreras-Moreira and Vinuesa, 2013). Out of 150,000 amino acid sequences in the Frankia pan genome, 420 proteins were identified as orthologs and part of the strict Frankia core genome. A concatenated phylogenetic tree was constructed. These concatenations were aligned using Clustal W (Larkin et al., 2007). The distance matrix was computed by JukesCantor method (Jukes and Cantor, 1969). The Neighboringjoining method (Saitou and Nei, 1987) was used to build the phylogeny. The percentage of replicate trees in which the associated taxa clustered together was determined using a bootstrap test (1000 replicates) (Felsenstein, 1985). Streptomyces coelicolor was used as an outgroup.

\section{RESULTS}

\section{CgRHD and CgNINA Activities Are Present in F. casuarinae Supernatant Fluid}

Previous studies have shown that factors inducing RHD in A. glutinosa (AgRHDF) are present in several Alnus-infective 

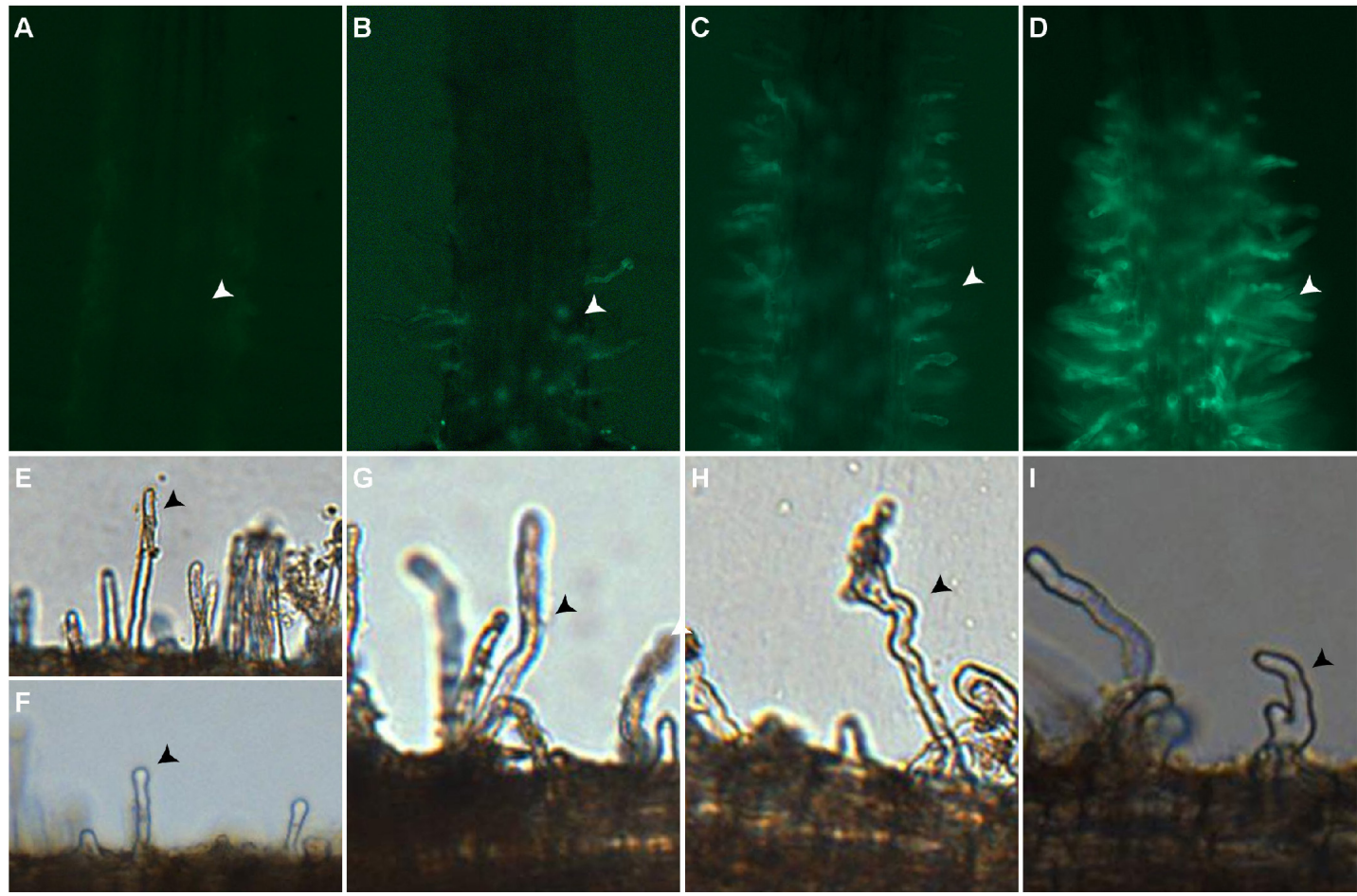

FIGURE 1 | Bioassays used to quantify the activation of ProCgNIN and root hair deformation in C. glauca. (A-D) Representative images showing levels of GFP fluorescence in root hairs of transgenic ProCgNIN:GFP C. glauca plants used for the CgNINA bioassay. Arrowheads indicate root hairs. (A) no signal, level 0; (B) weak signal, level 1; (C) medium signal, level 2; (D) strong signal, level 3. (E-I) representative images showing levels of root hair deformation in C. glauca used in the CgRHDF bioassay. (E) no deformation, level Oa; (F) tip swelling, level Ob; (G) one change in growth direction, level 1; (H) more than one change in growth direction but no bifurcation; level 2; (I) one or more bifurcations, level 3. Arrowheads indicate deformed root hairs.

Frankia strains (Prin and Rougier, 1987; Ghelue et al., 1997; Cérémonie et al., 1999). We recently found that $F$. casuarinae produces an extracellular factor, named CgNINA, which is able to induce the expression of the early symbiotic gene CgNIN in small lateral roots (Clavijo et al., 2015; Chabaud et al., 2016). To investigate whether a root hair deforming factor, hereafter named CgRHDF, was also produced by F. casuarinae, we incubated wild type C. glauca plants with $210^{-1}, 10^{-2} 10^{-3}$, and $10^{-4}$-fold dilutions of $F$. casuarinae supernatant fluids (FCS) and scored the deformation of root hairs situated in small lateral roots. In parallel, transgenic plants containing the ProCgNIN:GFP construct were incubated with the same solutions and the activation of ProCgNIN was recorded. As shown in Figure 2 and Supplementary Table S2, no RHD and no GFP fluorescence were detected in negative control roots treated with diluted BAP medium. RHD and GFP expression were maximal for the $10^{-2}$ dilution and lower levels of deformation and GFP fluorescence was observed with higher dilutions. The more concentrated dilution had a decreased response for both RHD and GFP fluorescence suggesting that the receptor could be saturated or inhibitory compounds may be associated with the extracts. At the dilutions $10^{-1}$ and $10^{-4}$, only the CgNINA bioassay showed a significant difference with the negative control suggesting that the CgNINA bioassay is more sensitive that the one based on CgRHD. The last three dilutions $\left(10^{-2}\right.$ to $\left.10^{-4}\right)$ appear to show dose-dependent responses for the CgNINA bioassay that may be used to quantify this factor.

\section{CgRHDF and CgNINA Share Physio-Chemical Properties}

CgRHDF and CgNINA properties were further compared by using similar treatments to those used to characterize CgNINA (Chabaud et al., 2016). First, the effects of temperature and $\mathrm{pH}$ sensitivity were analyzed. As shown in Figures $\mathbf{3 A}, \mathbf{B}$ and Supplementary Table S2, CgRHD was not affected by elevated temperatures (autoclaving) or treatment at $\mathrm{pH}$ values ranging from 5 to 10. However, cold treatment (freezing) or acidic $\mathrm{pH}$ conditions severely decreased CgRHD levels. Sonication of the inactivated fractions (frozen or acid-treated) resulted in a partial recovery of CgRHD activity (Figures $\mathbf{3 A}, \mathbf{B}$ and Supplementary Table S2). Similar results were obtained with the CgNINA bioassay. These results suggesting that both factors are thermoresistant and possibly precipitate at low $\mathrm{pH}$ or upon freezing but this aggregate can be resuspended using sonication. Both dialysis membranes and centrifugal filters were used to 


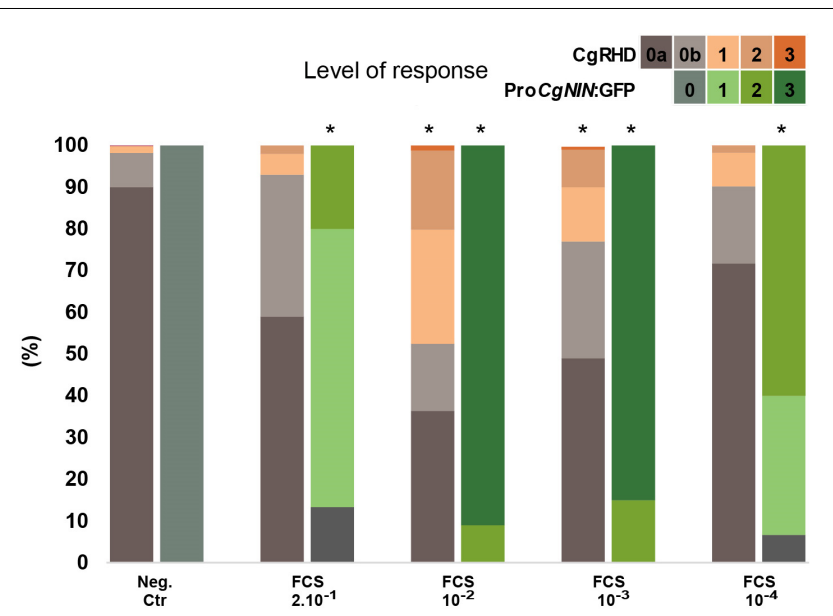

FIGURE 2 | Effect of cell-free $F$. casuarinae supernatant fluids (FCS) on Casuarina glauca root hair deformation (CgRHDF bioassay) and the activation of ProCgNIN (CgNINA bioassay). Plants were incubated with Frankia culture supernatant fluids (FCS) at the indicated dilutions and the Frankia culture medium BAP was used as a negative control. Orange bars represent the proportion of deformed root hairs in short lateral roots 2 days after contact with FCS dilutions. Green bars represent the proportion of plants expressing GFP in short lateral roots at different levels. Asterisks above bars indicate symbiotic responses significantly different from the negative control $(P<5 \%)$

determine the approximate size of CgRHDF and CgNINA. As shown in Figure 3C and Supplementary Table S2, CgRHDF and CgNINA were detected inside the 100-500 Da and the 500$1000 \mathrm{Da}$ cut-off dialysis tubing, but only a residual activity was found in the $3.5-5 \mathrm{kDa}$ ) suggesting that both factors correspond to small molecules with a molecular mass between 1 and $3.5 \mathrm{kDa}$. Experiments performed using centrifugal filters with 30, 10, and $3 \mathrm{kDa}$ cut-offs yielded similar results for the CgNINA bioassay (Figure 3D and Supplementary Table S2). However, maximum activity using the CgRHD bioassay was detected in the $3 \mathrm{kDa}$ and $10 \mathrm{kDa}$ retentates and only residual CgRHD was detected in the $3 \mathrm{kDa}$ flow though suggesting that both factors correspond to small but distinct molecules. Taken together these experiments suggest that the size of CgNINA is between 1 and $3 \mathrm{kDa}$ while CgRHDF is between 3 and $3.5 \mathrm{kDa}$. The polarity of the two factors was investigated using a butanol extraction. CgRHDF and CgNINA were only detected in the aqueous phase, indicating that both factors are hydrophilic (Figure $3 \mathrm{E}$ and Supplementary Table S2). Finally, we investigated whether CgRHDF contains a chitin backbone by performing the chitinase digestion experiment described in Chabaud et al. (2016). As shown in Figure 3F and Supplementary Table S2, the incubation with chitinase had no significant effect on CgRHDF or CgNINA activities. To rule out any inhibitory effect by FCS, we quantified the chitinase activity in the FCS/chitinase solution. As shown in Supplementary Figure S1, chitinase activity was not decreased by the addition of FCS to the chitinase solution. We conclude that F. casuarinae secretes two factors, CgNINA and CgRHDF, which are possibly two distinct molecules. Both factors sharing similar biochemical properties and correspond to small hydrophilic and thermoresistant molecules lacking a chitin backbone.

\section{Presence of RHDF and CgNINA in Frankia Strains Representative of Frankia Diversity}

We were interested in determining whether other Frankia strains had CgNINA and RHDF activities and tested different strains representing Frankia diversity. Cell-free supernatant fluids corresponding to 16 Frankia strains and another Actinobacteria, S. coelicolor, were tested for their capacity to deform C. glauca root hairs. As shown in Figure 4, Supplementary Table S3, and Supplementary Figure S2, CgRHD was present in all the Frankia belonging to clades I and III. The strongest activities were detected in strains that nodulate the genus Casuarina (clade Ic). Remarkably, RHD activity was also detected for several Frankia strains unable to form nodules on Casuarina such as F. alni (Clade Ia, Alnus-infective), Frankia eleagni and EANIpec (both clade III, Elaeagnus-infective strains). No deformation was detected for Frankia coriariae (clade II). Supernatant fluids from the atypical strains from clade IV or from the non-Frankia actinobacterium S. coelicolor did not induce RHD on Casuarina. Similar results were obtained when the same supernatant fluids were tested with the NIN bioassay (Figure 4, Supplementary Table S3, and Supplementary Figure S2) except for F. discariae.

The strong RHD and NINA activities obtained with the Alnusstrain $F$. alni prompted us to investigate whether the same culture supernatant fluids could induce RHD on A. glutinosa. As shown in Figure 4 and Supplementary Figure S2, responses observed in A. glutinosa were generally similar or stronger compared to the ones recorded in C. glauca particularly for strains belonging to group III. Interestingly, strains that were not able to activate ProCgNIN or to deform root hairs of C. glauca such as F. coriariae and the two atypical strains from group IV, EuI1c and CN3 were able to deform A. glutinosa root hairs. To confirm the absence of unintentional contaminations with a compatible strain, C. glauca, A. glutinosa, and O. trinervis were inoculated with the bacterial pellet obtained while preparing the supernatant fluids described above. As shown in Supplementary Table S3, nodules were only obtained with compatible strains, thus demonstrating that responses recorded with incompatible strains are not the result of any contamination; incompatible strains are thus probably able to synthetize CgRHDF and CgNINA.

Surprisingly for F. discariae, we detected not only weak levels of RHD in C. glauca but also a weak activation of ProCgNIN and a strong deformation of root hairs in A. glutinosa (Figure 4 and Supplementary Figure S2). These results are in apparent contradiction with our previous work, in which we could not detect any activation of ProCgNIN in response to $F$. discariae supernatants. However, at that time $F$. discariae cultures were induced with RE from O. trinervis instead of C. glauca (Chabaud et al., 2016). We therefore repeated the experiments for F. alni and $F$. discariae using RE from A. glutinosa and O. trinervis, respectively. As shown in Figure 4, Supplementary Table S3, and Supplementary Figure S2, incubation of $F$. alni with RE from the host plant (A. glutinosa) did not change the response of C. glauca, but slightly increased the response of A. glutinosa. For $F$. discariae, incubation with exudates from $O$. trinervis reduced the level of ProCgNIN activation compared to the 

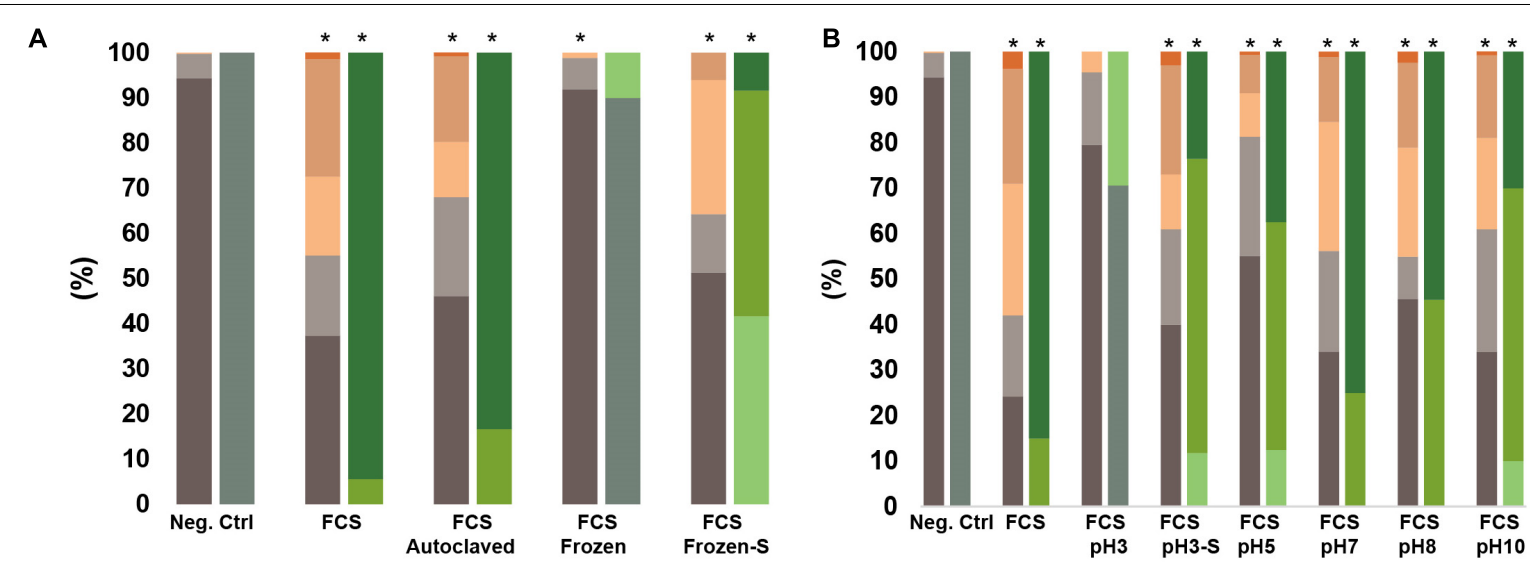

C

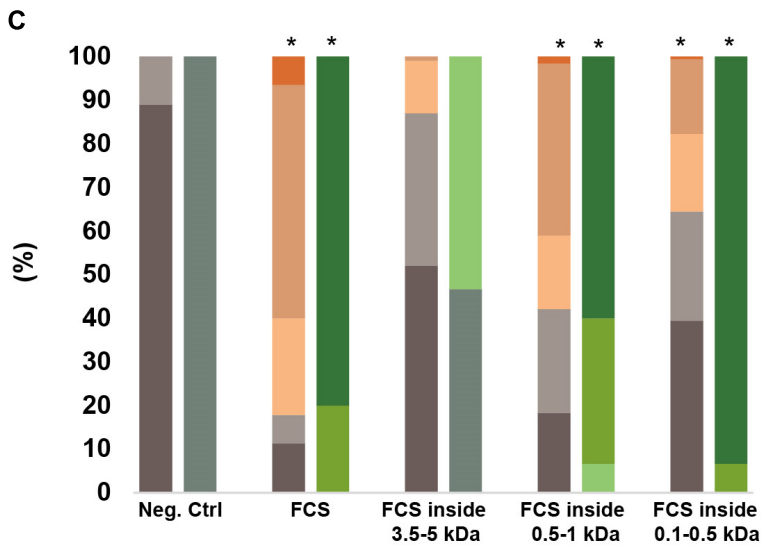

D

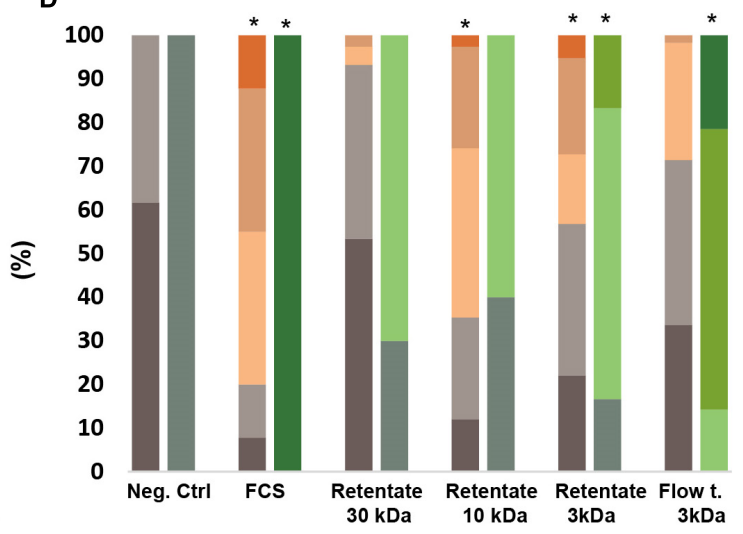

E
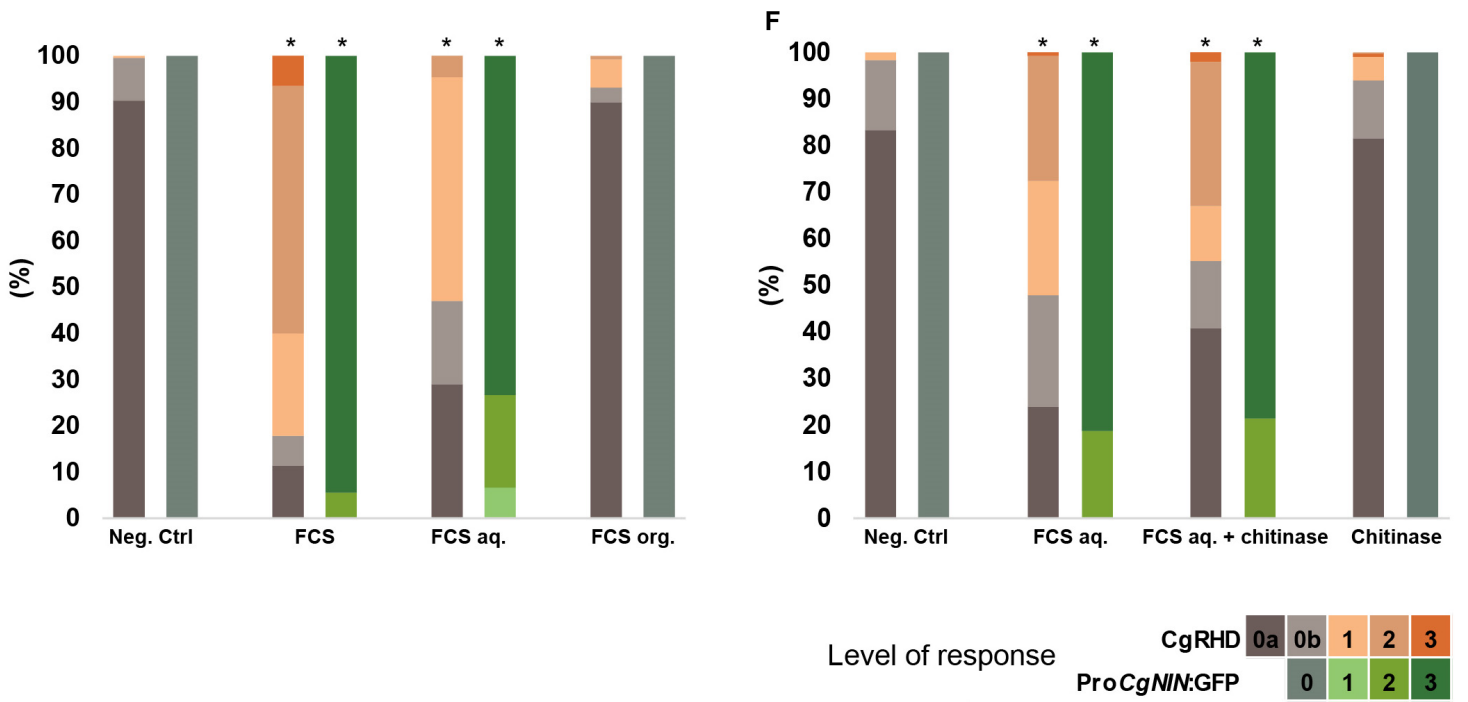

FIGURE 3|Physico-chemical properties of CgRHDF and CgNINA. Orange bars show the proportion of deformed root hairs in short lateral roots 2 days after contact with FCS submitted to the different treatments. BAP medium diluted 100 times was used as a negative control. Asterisks above bars indicate symbiotic responses significantly different from the negative control $(P<5 \%)$. (A) Temperature sensitivity. High levels of CgRHD and GFP were detected in autoclaved FCS but no significant activity was present in FCS that was previously frozen. Sonication of frozen FCS (Frozen-S) allowed partial recovery of CgRHD and CgNINA activities. (B) $\mathrm{pH}$ sensitivity: FCS were incubated at different $\mathrm{pH}$ for one hour at the indicated $\mathrm{pH}$. Sonication of FCS incubated at $\mathrm{pH} 3(\mathrm{pH} 3-\mathrm{S})$ restored CgRHD and CgNINA levels similar to the untreated control. (C) Size estimation using a dialysis tubing. CgRHD and CgNINA activities inside dialysis tubings with the indicated cutoffs was scored (D) Size estimation using centrifugal filters. FCS were submitted to successive filtrations using filters with the indicated cut-offs. CgRHD and CgNINA were evaluated on the retentate or the flow through (Flow t.). (E) CgRHD and CgNINA activity after 1-butanol extraction. Significant activities were only detected in the aqueous fraction (FCSaq) and not in the organic fraction (FCSorg). (F) Sensitivity to Chitinase. FCSaq incubated with chitinase showed similar CgRHD and CgNINA activities compared to untreated FCS. 


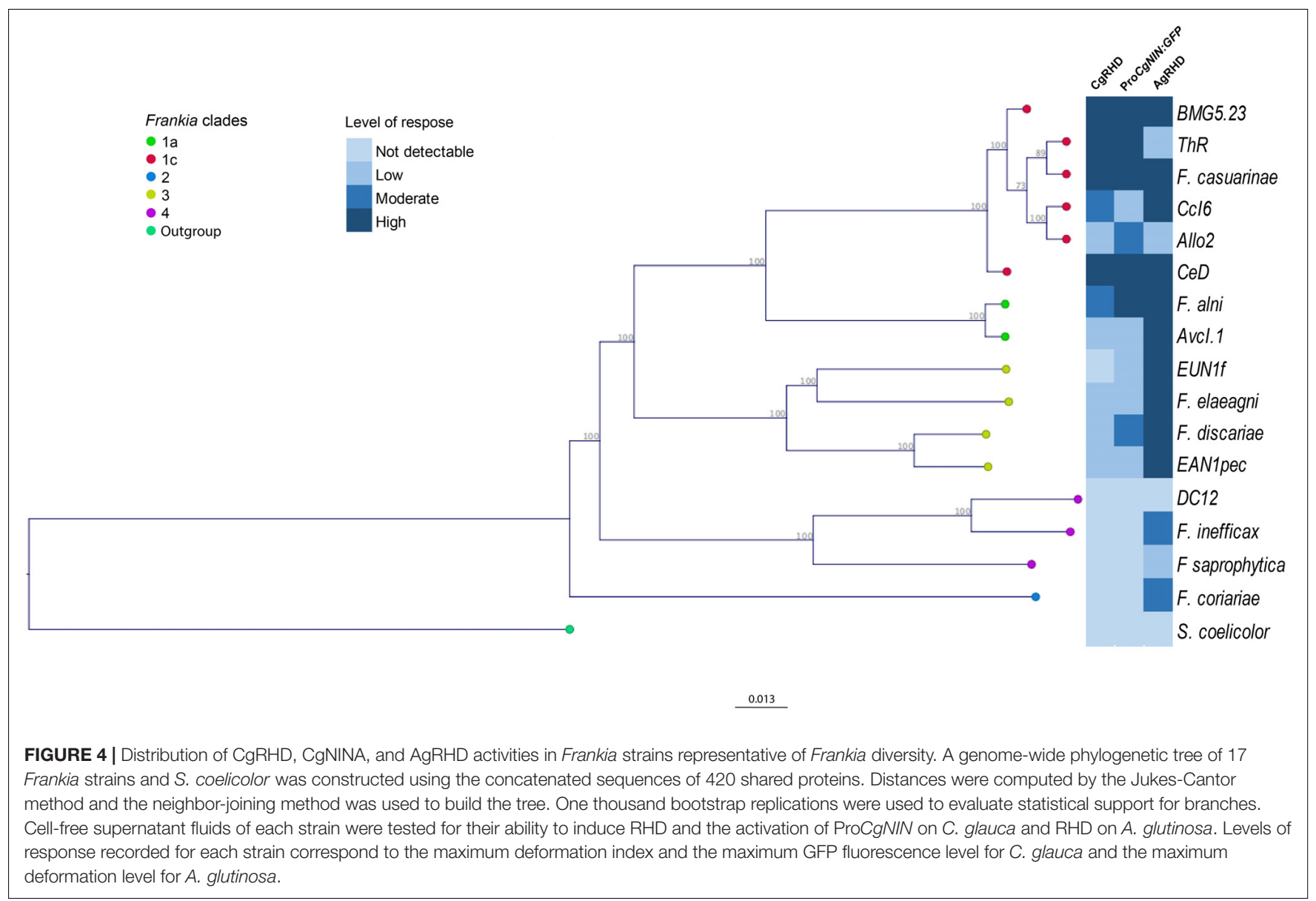

experiment performed with RE from C. glauca or without RE and the results obtained were not significantly different from the negative control (Supplementary Figure S2). Together, these results showed that $F$. discariae is able to synthetize molecules able to induce CgRHD, AgRHD, and the activation of ProCgNIN. The activation of ProCgNIN was enhanced when $F$. discariae was incubated with RE from C. glauca and was possibly below detection limits using the less sensitive equipment described in (Chabaud et al., 2016).

\section{DISCUSSION}

\section{Presence of CgRHDF and Comparison to CgNINA}

In legumes, RHD is one of the earliest visible responses induced upon recognition of rhizobial NFs by the host plant, and the development of a bioassay based on RHD was crucial to identify the chemical nature of NFs (Lerouge et al., 1990). The perception of NFs also provokes significant alterations of gene expression and notably the expression of symbiosisinduced genes such as MtEnod11 (Journet et al., 2001) and NIN (Schauser et al., 1999; Radutoiu et al., 2003). In actinorhizal plants infected intracellularly such as C. glauca and A. glutinosa, RHD is also one of the first visible responses to Frankia inoculation and factors able to induce RHD in Alnus (AgRHDF) have been partially purified and characterized (Cérémonie et al., 1999). In C. glauca, we have used transgenic plants expressing a ProCgNIN:GFP fusion to characterize CgNINA, a factor present in cell free $F$. casuarinae supernatant fluids able to activate the CgNIN promoter in C. glauca root hairs. Here we have shown that a CgRHDF is also present in cellfree F. casuarinae supernatant fluids. Furthermore, we have shown that CgRHDF and CgNINA share similar physicochemical properties listed in Table 1. Interestingly the experiment performed with centrifugal filters suggests that CgNINA and CgRHDF are both small molecules with slightly different sizes, CgNINA being probably smaller than CgRHDF. This observation is intriguing if CgNINA or CgRHDF are the actinorhizal analogs of rhizobial NFs because NFs are known to induce both RHD and the expression of early nodulins such as NIN. Responses obtained with centrifugal filters were however, less contrasted compared to the other experiments shown here and residual CgRHD activity was still present in the $3 \mathrm{kDa}$ flow through. We therefore cannot exclude that CgNINA also possess a small RHD activity and additional experiments are needed to confirm this hypothesis. If CgRHDF and CgNINA can indeed be separated, it would be interesting to know if those molecules are able to induce the high frequency nuclear $\mathrm{Ca}^{2}+$ spiking in growing C. glauca root hairs as described previously (Chabaud et al., 
TABLE 1 | Properties of CgRHDF, CgNINA, AgRHDF, and rhizobial NFs.

\begin{tabular}{|c|c|c|c|c|}
\hline & CgRHDF & CgNINA & AgRHDF & Rhizobial NF \\
\hline \multirow[t]{2}{*}{ Induction } & Inducible & Inducible & Inducible & Inducible \\
\hline & (root exudates) & (root exudates) & (root exudates) & (flavonoids) \\
\hline Size & $1-3 \mathrm{kDa}$ & $1-3 \mathrm{kDa}$ & $1.2-3 \mathrm{kDa}$ & $1 \mathrm{kDa}$ \\
\hline Thermal stability & Thermoresistant & Thermoresistant & Thermoresistant & Thermoresistant \\
\hline Active concentration & $10^{-2}-10^{-3}$ (supernatant) & $10^{-1}-10^{-4}$ (supernatant) & $10^{-3} 10^{-5}$ (supernatant) & Down to $10^{-12} \mathrm{M}$ \\
\hline Hydrophilicity & Hydrophilic & Hydrophilic & Hydrophilic & Amphiphilic \\
\hline Chitinase action & resistant & resistant & resistant & sensitive \\
\hline
\end{tabular}

2016). The ability to induce $\mathrm{Ca}^{2}+$ oscillations in response to symbiotic bacteria is a common feature of nodulating species within the nitrogen-fixing clade (Granqvist et al., 2015). Alternatively we hypothesize that CgRHDF and CgNINA are the same molecule but a cofactor or a specific decoration is needed to enhance CgRHD activity without affecting its ability to activate ProCgNIN. The $3 \mathrm{kDa}$ centrifugal filter possibly eliminated decorated molecules with higher mass or cofactors and therefore strong activity was only detected with the CgNINA bioassay.

\section{Comparison With AgRHDF and Rhizobial Nod Factors}

CgRHDF and CgNINA also share many characteristics with AgRHDF, the corresponding factor characterized using A. glutinosa/F. alni (Table 1; Ghelue et al., 1997; Cérémonie et al., 1999). However, both factors appear to be structurally different from the rhizobial NFs because unlike NFs they are not found in the organic phase following a butanol extraction and are not sensitive to the endochitinase from Aeromonas hydrophila (Cérémonie et al., 1999) or the exochitinase from S. griseus (Cérémonie et al., 1999; Chabaud et al., 2016). This difference is in agreement with (1) the lack of $\operatorname{nodA}$ genes in the sequenced genomes of $F$. casuarinae and F. alni (Normand et al., 2007). (2) the absence of chitin oligomers in F. alni supernatant fluids (Cérémonie et al., 1999), and (3) the failure of NFs from the broad host-range rhizobia NGR234 to elicit RHD or $\mathrm{Ca}^{2}+$ spiking in A. glutinosa or C. glauca (Cérémonie et al., 1999; Granqvist et al., 2015; Chabaud et al., 2016). The possibility that actinorhizal recognition is mediated by molecules that are not hydrolyzed by tested chitinases is unexpected because downstream components of the NF signaling pathway are conserved not only between actinorhizal and rhizobial symbioses (Svistoonoff et al., 2014; Griesmann et al., 2018), but also between rhizobial and arbuscular-mycorrhizal symbioses where chitin-derived MycLCOs and COs play an important role as signaling molecules (Camps et al., 2015). Putative orthologs of NF receptors are present in C. glauca and A. glutinosa (Hocher et al., 2011). We are currently studying whether these genes play a role in actinorhizal symbioses. Because LysM receptor kinases have been shown to recognize not only chitin-derived molecules but also peptidoglycans and exopolysaccharides (Willmann et al., 2011; Kawaharada et al., 2015), orthologs of genes encoding NF receptors could be involved in the recognition of
CgRHDF/NINA or AgRHDF, even if their chemical backbone is not chitin-based.

\section{Presence of NINA and CgRHDF in Other Frankia Strains}

In most legumes, NFs allow the specific recognition between the host plant and its symbiotic rhizobia (Masson-Boivin et al., 2009; Oldroyd, 2013). Changes in specific decorations often result in host incompatibility (Dénarié et al., 1996) but NFs from incompatible strains can induce symbiotic responses such as RHD and activation of symbiotic genes when applied at increased concentrations (Roche et al., 1991). These results can be explained by changes of affinity between NFs and the cognate NF receptors able to recognize the chitin backbone and also the modified backbone structure. A misrecognition leads to decreased affinity but this can be compensated by increased amounts of substrate (Dénarié et al., 1996). The symbiotic responses in non-host plants reported here point to a similar mechanism in C. glauca: strains from clades I and III possibly synthesize molecules sharing a common molecular backbone that is recognized by $C$. glauca receptors inducing RHD and the activation of ProCgNIN promoter. Optimal recognition is achieved for compatible strains (clade Ic) and some related strains (F. alni from clade Ia) but only the backbone would be recognized for more distant strains (clade III). This recognition is not detectable for non-infective strains (clade IV) and the distantly related strain $F$. coriariae suggesting that those strains do not produce sufficient amounts of this recognized backbone under the tested conditions.

\section{Comparison Between CgRHDF and AgRHDF}

Compared to CgRHDF and CgNINA, the distribution of AgRHDF seems less related to phylogeny. Generally, AgRHDF levels were stronger for clade III strains and several strains without any CgRHDF or CgNINA activity ( $F$. coriariae and two uninfective strains from clade IV). These differences suggest that the AgRHDF assay detects smaller concentrations of deforming factors or that root hairs of A. glutinosa are deformed by a wider range of molecules compared to C. glauca. This second hypothesis is in agreement with RHD detected in Alnus roots incubated with non-Frankia bacteria or fungi (Berry and Torrey, 1983; Knowlton and Dawson, 1983; Prin and Rougier, 1987; Sequerra et al., 1994). 


\section{Impact of Root Exudates}

We also found that the nature of RE used to incubate Frankia cultures could have an impact on RHDF and CgNINA activities. Unexpectedly lower CgRHDF and CgNINA activities were found when $F$. discariae was cultivated with $\mathrm{RE}$ from $O$. trinervis compared to RE from C. glauca. In legumes, specific flavonoids secreted by the host plant induce the expression of nod genes and the synthesis of NFs (Oldroyd, 2013). RE secreted by the host plant probably also play a role in actinorhizae formation because the incubation with RE induces morphological changes in Frankia and accelerate the nodulation process (Gabbarini and Wall, 2008, 2011; Beauchemin et al., 2012). Myricaceae seed extracts also influence Frankia growth (Bagnarol et al., 2007). In Alnus AgRHDF is reported to be produced either constitutively (McEwan et al., 1992; Ghelue et al., 1997; Cérémonie et al., 1999) or upon induction with RE (Prin and Rougier, 1987). Different plant RE have different effects on Frankia physiology. Information about CgRHDF is scarce but flavonoids isolated from Casuarina seeds have been shown to induce the production of CgRHDF by the Casuarina-infective BR strain (Selim, 1995). Increased CgRHD activity in F. discariae incubated with C. glauca $\mathrm{RE}$ could be due to increased amounts of flavonoids in Casuarina $\mathrm{RE}$ compared to O. trinervis.

\section{AUTHOR CONTRIBUTIONS}

MC, VH, PP, MS, and SSv conceived and designed the experiments. $\mathrm{MC}, \mathrm{PP}, \mathrm{PF}, \mathrm{VH}$, and $\mathrm{AC}-\mathrm{M}$ performed the

\section{REFERENCES}

Alloisio, N., Queiroux, C., Fournier, P., Pujic, P., Normand, P., Vallenet, D., et al. (2010). The Frankia alni symbiotic transcriptome. Mol. Plant. Microbe Interact. 23, 593-607. doi: 10.1094/MPMI-23-5-0593

Bagnarol, E., Popovici, J., Alloisio, N., Maréchal, J., Pujic, P., Normand, P., et al. (2007). Differential Frankia protein patterns induced by phenolic extracts from Myricaceae seeds. Physiol. Plant. 130, 380-390. doi: 10.1111/j.1399-3054.2007. 00875.x

Beauchemin, N. J., Furnholm, T., Lavenus, J., Svistoonoff, S., Doumas, P., Bogusz, D., et al. (2012). Casuarina root exudates alter the physiology, surface properties, and plant infectivity of Frankia sp. strain CcI3. Appl. Environ. Microbiol. 78, 575-580. doi: 10.1128/AEM.06183-11

Benson, D. R., Brooks, J. M., Huang, Y., Bickhart, D. M., and Mastronunzio, J. E. (2011). The biology of Frankia sp. strains in the post-genome Era. Mol. Plant. Microbe Interact. 24, 1310-1316. doi: 10.1094/MPMI-06-11-0150

Berry, A. M., and Torrey, J. G. (1983). Root hair deformation in the infection process of Alnus rubra. Can. J. Bot. 61, 2863-2876. doi: 10.1139/b83-319

Camps, C., Jardinaud, M.-F., Rengel, D., Carrère, S., Hervé, C., Debellé, F., et al. (2015). Combined genetic and transcriptomic analysis reveals three major signalling pathways activated by Myc-LCOs in Medicago truncatula. New Phytol. 208, 224-240. doi: 10.1111/nph.13427

Cérémonie, H., Cournoyer, B., Maillet, F., Normand, P., and Fernandez, M. P. (1998). Genetic complementation of rhizobial nod mutants with Frankia DNA: artifact or reality? Mol. Gen. Genet. MGG 260, 115-119. doi: 10.1007/ s004380050877

Cérémonie, H., Debellé, F., and Fernandez, M. P. (1999). Structural and functional comparison of Frankia root hair deforming factor and rhizobia Nod factor. Can. J. Bot. 77, 1293-1301. doi: 10.1139/b99-060

Chabaud, M., Gherbi, H., Pirolles, E., Vaissayre, V., Fournier, J., Moukouanga, D., et al. (2016). Chitinase-resistant hydrophilic symbiotic factors secreted by experiments. MC, SSv, PP, PF, and VH analyzed the data. SS and MC statistical analyzed the data. MS, MG, MC, VH, HG, DG, AC-M, SP, AC, MN, ES, CP, and SSv contributed reagents, materials, analysis, and tools. MC, SSv, and LT wrote the paper. All authors read the final version of the manuscript.

\section{FUNDING}

This work was supported by IRD (French National Research Institute for Sustainable Development), and the United States Department of Agriculture (project USDA NIFA 2015-6701422849).

\section{ACKNOWLEDGMENTS}

We would like to thank Maurice Sagna (UCAD, Dakar) for his help with FCS concentration, L. Wall (Quilmes University, Argentina) and E. Chaia (U. Comahue, Argentina) for providing O. trinervis seeds.

\section{SUPPLEMENTARY MATERIAL}

The Supplementary Material for this article can be found online at: https://www.frontiersin.org/articles/10.3389/fpls.2018.01494/ full\#supplementary-material

Frankia activate both Ca2 + spiking and NIN gene expression in the actinorhizal plant Casuarina glauca. New Phytol. 209, 86-93. doi: 10.1111/nph. 13732

Clavijo, F., Diedhiou, I., Vaissayre, V., Brottier, L., Acolatse, J., Moukouanga, D., et al. (2015). The Casuarina NIN gene is transcriptionally activated throughout Frankia root infection as well as in response to bacterial diffusible signals. New Phytol. 208, 887-903. doi: 10.1111/nph.13506

Contreras-Moreira, B., and Vinuesa, P. (2013). GET_Homologues, a versatile software package for scalable and robust microbial pangenome analysis. Appl. Environ. Microbiol. 79, 7696-7701. doi: 10.1128/AEM.02411-13

Dawson, J. O. (eds). (2008). "Ecology of actinorhizal plants," in Nitrogen-fixing Actinorhizal Symbioses (Berlin: Springer), 199-234. doi: 10.1007/978-1-40203547-0_8

Dénarié, J., Débelle, F., and Prome, J.-C. (1996). Rhizobium lipochitooligosaccharide nodulation factors: signaling molecules mediating recognition and morphogenesis. Annu. Rev. Biochem. 65, 503-535. doi: 10.1146/annurev.bi.65.070196.002443

Fahraeus, G. (1957). The infection of clover root hairs by nodule bacteria studied by a simple glass slide technique. Microbiology 16, 374-381. doi: 10.1099/ 00221287-16-2-374

Felsenstein, J. (1985). Confidence limits on phylogenies: an approach using the bootstrap. Evolution 39, 783-791. doi: 10.1111/j.1558-5646.1985.tb00420.x

Gabbarini, L., and Wall, L. (2008). Analysis of nodulation kinetics in FrankiaDiscaria trinervis symbiosis reveals different factors involved in the nodulation process. Physiol. Plant. 133, 776-785. doi: 10.1111/j.1399-3054.2008.01096.x

Gabbarini, L., and Wall, L. (2011). Diffusible factors involved in early interactions of actinorhizal symbiosis are modulated by the host plant but are not enough to break the host range barrier. Funct. Plant Biol. 38, 671-681. doi: 10.1071/ FP11003

Ghelue, M. V., Løvaas, E., Ringø, E., and Solheim, B. (1997). Early interactions between Alnus glutinosa and Frankia strain ArI3. Production and specificity 
of root hair deformation factor (s). Physiol. Plant. 99, 579-587. doi: 10.1111/j. 1399-3054.1997.tb05360.x

Granqvist, E., Sun, J., Op den Camp, R., Pujic, P., Hill, L., Normand, P., et al. (2015). Bacterial-induced calcium oscillations are common to nitrogen-fixing associations of nodulating legumes and nonlegumes. New Phytol. 207, 551-558. doi: 10.1111/nph.13464

Griesmann, M., Chang, Y., Liu, X., Song, Y., Haberer, G., Crook, M. B., et al. (2018). Phylogenomics reveals multiple losses of nitrogen-fixing root nodule symbiosis. Science 361:eaat1743. doi: 10.1126/science.aat1743

Hocher, V., Alloisio, N., Auguy, F., Fournier, P., Doumas, P., Pujic, P., et al. (2011). Transcriptomics of actinorhizal symbioses reveals homologs of the whole common symbiotic signaling cascade. Plant Physiol. 156, 700-711. doi: 10.1104/pp.111.174151

Hocher, V., Auguy, F., Argout, X., Laplaze, L., Franche, C., and Bogusz, D. (2006). Expressed sequence-tag analysis in Casuarina glauca actinorhizal nodule and root. New Phytol. 169, 681-688. doi: 10.1111/j.1469-8137.2006.01644.x

Imanishi, L., Vayssières, A., Franche, C., Bogusz, D., Wall, L., and Svistoonoff, S. (2011). Transformed hairy roots of Discaria trinervis: a valuable tool for studying actinorhizal symbiosis in the context of intercellular infection. Mol. Plant. Microbe Interact. 24, 1317-1324. doi: 10.1094/MPMI-03-11-0078

Journet, E. P., El-Gachtouli, N., Vernoud, V., de Billy, F., Pichon, M., Dedieu, A., et al. (2001). Medicago truncatula ENOD11: a novel RPRP-encoding early nodulin gene expressed during mycorrhization in arbuscule-containing cells. Mol. Plant. Microbe Interact. 14, 737-748. doi: 10.1094/MPMI.2001.14.6.737

Jukes, T. H., and Cantor, C. R. (1969). Evolution of Protein Molecules. Cambridge, MA: New York Academic Press, 21-132.

Kawaharada, Y., Kelly, S., Nielsen, M. W., Hjuler, C. T., Gysel, K., Muszyński, A., et al. (2015). Receptor-mediated exopolysaccharide perception controls bacterial infection. Nature 523, 308-312. doi: 10.1038/nature14611

Knowlton, S., and Dawson, J. O. (1983). Effects of Pseudomonas cepacia and cultural factors on the nodulation of Alnus rubra roots by Frankia. Can. J. Bot. 61, 2877-2882. doi: 10.1139/b83-320

Ktari, A., Gueddou, A., Nouioui, I., Miotello, G., Sarkar, I., Ghodhbane-Gtari, F., et al. (2017). Host plant compatibility shapes the proteogenome of Frankia coriariae. Front. Microbiol. 8:720. doi: 10.3389/fmicb.2017.00720

Kucho, K., Kakoi, K., Yamaura, M., Higashi, S., Uchiumi, T., and Abe, M. (2009). Transient transformation of Frankia by fusion marker genes in liquid culture. Microbes Environ. 24, 231-240. doi: 10.1264/jsme2.ME09115

Kucho, K.-I., Tamari, D., Matsuyama, S., Nabekura, T., and Tisa, L. S. (2017). Nitrogen fixation mutants of the actinobacterium Frankia casuarinae CcI3. Microbes Environ. 32, 344-351. doi: 10.1264/jsme2.ME17099

Larkin, M. A., Blackshields, G., Brown, N. P., Chenna, R., McGettigan, P. A., McWilliam, H., et al. (2007). Clustal W and clustal X version 2.0. Bioinformatics 23, 2947-2948. doi: 10.1093/bioinformatics/btm404

Lerouge, P., Roche, P., Faucher, C., Maillet, F., Truchet, G., Promé, J. C., et al. (1990). Symbiotic host-specificity of Rhizobium meliloti is determined by a sulphated and acylated glucosamine oligosaccharide signal. Nature 344, 781784. doi: $10.1038 / 344781 \mathrm{a} 0$

Lhuissier, F. G. P., De Ruijter, N. C. A., Sieberer, B. J., Esseling, J. J., and Emons, A. M. C. (2001). Time course of cell biological events evoked in legume root hairs by rhizobium Nod Factors: state of the art. Ann. Bot. 87, 289-302. doi: 10.1006/anbo.2000.1333

Marsh, J. F., Rakocevic, A., Mitra, R. M., Brocard, L., Sun, J., Eschstruth, A., et al. (2007). Medicago truncatula NIN is essential for rhizobial-independent nodule organogenesis induced by autoactive calcium/calmodulindependent protein kinase. Plant Physiol. 144, 324-335. doi: 10.1104/pp.106. 093021

Masson-Boivin, C., Giraud, E., Perret, X., and Batut, J. (2009). Establishing nitrogen-fixing symbiosis with legumes: how many rhizobium recipes? Trends Microbiol. 17, 458-466. doi: 10.1016/j.tim.2009.07.004

Mastronunzio, J. E., and Benson, D. R. (2010). Wild nodules can be broken: proteomics of Frankia in field-collected root nodules. Symbiosis 50, 13-26. doi: 10.1007/s13199-009-0030-1

McEwan, N. R., Green, D. C., and Wheeler, C. T. (1992). Utilisation of the root hair curling reaction in Alnus glutinosa for the assay of nodulation signal compounds. Acta Oecol. 13, 509-510.

Ngom, M., Gray, K., Diagne, N., Oshone, R., Fardou, J., Gherbi, H., et al. (2016). Performance of diverse Frankia strains on salt-stressed Casuarina glauca and
Casuarina equisetifolia Plants. Front. Plant Sci. 7:1331. doi: 10.3389/fpls.2016. 01331

Nguyen, T. V., Wibberg, D., Battenberg, K., Blom Brian, J. B., Heuvel, V., Berry, A. M., et al. (2016). An assemblage of Frankia Cluster II strains from California contains the canonical nod genes and also the sulfotransferase gene nodH. BMC Genemics 17:796. doi: 10.1186/s12864-016-3140-1

Normand, P., Lapierre, P., Tisa, L. S., Gogarten, J. P., Alloisio, N., Bagnarol, E., et al. (2007). Genome characteristics of facultatively symbiotic Frankia sp. strains reflect host range and host plant biogeography. Genome Res. 17, 7-15. doi: $10.1101 /$ gr.5798407

Oldroyd, G. E. D. (2013). Speak, friend, and enter: signalling systems that promote beneficial symbiotic associations in plants. Nat. Rev. Microbiol. 11, 252-263. doi: $10.1038 /$ nrmicro2990

Parham, J. A., and Deng, S. P. (2000). Detection, quantification and characterization of $\beta$-glucosaminidase activity in soil. Soil Biol. Biochem. 32, 1183-1190. doi: 10.1016/S0038-0717(00)00034-1

Perrine-Walker, F., Gherbi, H., Imanishi, L., Hocher, V., GhodhbaneGtari, F., Lavenus, J., et al. (2011). Symbiotic signaling in actinorhizal symbioses. Curr. Protein Pept. Sci. 12, 156-164. doi: 10.2174/1389203117956 84896

Persson, T., Battenberg, K., Demina, I. V., Vigil-Stenman, T., Vanden Heuvel, B., Pujic, P., et al. (2015). Candidatus Frankia Datiscae Dg1, the actinobacterial microsymbiont of Datisca glomerata, expresses the canonical nod genes nodABC in symbiosis with its host plant. PLoS One 10:e0127630. doi: 10.1371/ journal.pone. 0127630

Prin, Y., and Rougier, M. (1987). Preinfection events in the establishment of Alnusfrankia symbiosis: study of the root hair deformation step. Life Sci. Adv. 6, 98-106.

R Core Team (2013). R: A Language and Environment for Statistical Computing. Vienna: R Core Team.

Radutoiu, S., Madsen, L. H., Madsen, E. B., Felle, H. H., Umehara, Y., Grønlund, M., et al. (2003). Plant recognition of symbiotic bacteria requires two LysM receptor-like kinases. Nature 425, 585-592. doi: 10.1038/nature02039

Roche, P., Debellé, F., Maillet, F., Lerouge, P., Faucher, C., Truchet, G., et al. (1991). Molecular basis of symbiotic host specificity in Rhizobium meliloti: nodH and nodPQ genes encode the sulfation of lipo-oligosaccharide signals. Cell 67, 1131-1143. doi: 10.1016/0092-8674(91)90290-F

Saitou, N., and Nei, M. (1987). The neighbor-joining method: a new method for reconstructing phylogenetic trees. Mol. Biol. Evol. 4, 406-425. doi: 10.1093/ oxfordjournals.molbev.a040454

Schauser, L., Roussis, A., Stiller, J., and Stougaard, J. (1999). A plant regulator controlling development of symbiotic root nodules. Nature 402, 191-195. doi: $10.1038 / 46058$

Selim, S. M. A. (1995). La Symbiose Casuarina-Frankia. Optimisation de la Croissance et Approche Biologique de la Reconnaissance Microorganisme-Plante Hote. Available at: http://www.theses.fr/1995PA112288 [accessed February 27, 2018].

Sequerra, J., Capellano, A., Faure-Raynard, M., and Moiroud, A. (1994). Root hair infection process and myconodule formation on Alnus incana by Penicillium nodositatum. Can. J. Bot. 72, 955-962. doi: 10.1139/b94-121

Soyano, T., Hirakawa, H., Sato, S., Hayashi, M., and Kawaguchi, M. (2014). NODULE INCEPTION creates a long-distance negative feedback loop involved in homeostatic regulation of nodule organ production. Proc. Natl. Acad. Sci. U.S.A. 111, 14607-14612. doi: 10.1073/pnas.14127 16111

Soyano, T., Kouchi, H., Hirota, A., and Hayashi, M. (2013). NODULE INCEPTION directly targets NF-Y subunit genes to regulate essential processes of root nodule development in Lotus japonicus. PLoS Genet. 9:e1003352. doi: 10.1371/ journal.pgen.1003352

Svistoonoff, S., Gherbi, H., Nambiar-Veetil, M., Zhong, C., Michalak, Z., Laplaze, L., et al. (2010a). Contribution of transgenic Casuarinaceae to our knowledge of the actinorhizal symbioses. Symbiosis 50, 3-11. doi: 10.1007/ s13199-009-0036-8

Svistoonoff, S., Sy, M. O., Diagne, N., Barker, D. G., Bogusz, D., and Franche, C. (2010b). Infection-specific activation of the Medicago truncatula Enod11 early nodulin gene promoter during actinorhizal root nodulation. Mol. Plant. Microbe Interact. 23, 740-747. doi: 10.1094/MPMI-23-60740 
Svistoonoff, S., Hocher, V., and Gherbi, H. (2014). Actinorhizal root nodule symbioses: what is signalling telling on the origins of nodulation? Curr. Opin. Plant Biol. 20, 11-18. doi: 10.1016/j.pbi.2014.03.001

Tisa, L. S., Oshone, R., Sarkar, I., Ktari, A., Sen, A., and Gtari, M. (2016). Genomic approaches toward understanding the actinorhizal symbiosis: an update on the status of the Frankia genomes. Symbiosis 70, 5-16. doi: 10.1007/s13199-0160390-2

Vessey, K. J., Pawlowski, K., and Bergman, B. (2005). Root-based N 2fixing symbioses: legumes, actinorhizal plants, Parasponia sp. and cycads. Root Physiol. Gene Funct. 266, 51-78. doi: 10.1007/1-40204099-7_3

Wall, L. G. (2000). The actinorhizal symbiosis. J. Plant Growth Regul. 19, 167-182.

Willmann, R., Lajunen, H. M., Erbs, G., Newman, M.-A., Kolb, D., Tsuda, K., et al. (2011). Arabidopsis lysin-motif proteins LYM1 LYM3 CERK1 mediate bacterial peptidoglycan sensing and immunity to bacterial infection. Proc. Natl. Acad. Sci. U.S.A. 108, 19824-19829. doi: 10.1073/pnas.11128 62108
Yoro, E., Suzaki, T., Toyokura, K., Miyazawa, H., Fukaki, H., and Kawaguchi, M. (2014). a positive regulator of nodule organogenesis, nodule inception, acts as a negative regulator of rhizobial infection in Lotus japonicus. Plant Physiol. 165, 747-758. doi: 10.1104/pp.113.233379

Conflict of Interest Statement: The authors declare that the research was conducted in the absence of any commercial or financial relationships that could be construed as a potential conflict of interest.

Copyright (C) 2018 Cissoko, Hocher, Gherbi, Gully, Carré-Mlouka, Sane, Pignoly, Champion, Ngom, Pujic, Fournier, Gtari, Swanson, Pesce, Tisa, Sy and Svistoonoff. This is an open-access article distributed under the terms of the Creative Commons Attribution License (CC BY). The use, distribution or reproduction in other forums is permitted, provided the original author(s) and the copyright owner(s) are credited and that the original publication in this journal is cited, in accordance with accepted academic practice. No use, distribution or reproduction is permitted which does not comply with these terms. 